\title{
Low-mass variable stars in the globular cluster NGC 6397
}

\author{
E. Martinazzi, ${ }^{1,2 \star}$ S. O. Kepler ${ }^{1 \star}$ and J. E. S. Costa ${ }^{1 \star}$ \\ ${ }^{1}$ Instituto de Física, Universidade Federal do Rio Grande do Sul, 91501-900 Porto Alegre, RS, Brazil \\ ${ }^{2}$ Instituto Federal do Rio Grande do Sul, 95700-000 Bento Gonçalves, RS, Brazil
}

Accepted 2017 February 26. Received 2017 February 26; in original form 2015 December 1

\begin{abstract}
We have conducted a photometric survey of the globular cluster NGC 6397 in a search for variable stars. We obtained $\sim 11 \mathrm{~h}$ of time-resolved photometric images with one European Southern Observatory-Very Large Telescope using the FOcal Reducer and low dispersion Spectrograph imager distributed over two consecutive nights. We analysed 8391 light curves of stars brighter than magnitude 23 with the $465-\mathrm{nm}$ filter, and we identified 412 variable stars, reaching $\sim 4.8 \pm 0.2$ per cent of variability with time-scales between 0.004 and $2 \mathrm{~d}$, with amplitudes variation greater than \pm 0.2 mag.
\end{abstract}

Key words: convection - methods: data analysis - techniques: photometric-stars: chromospheres - stars: low-mass - stars: variables: general.

\section{INTRODUCTION}

Stellar variability depends on effective temperature, magnetic field and also on the opacity, which is metallicity-dependent. Globular clusters offer a way to study this dependence. As globular clusters are some of the oldest objects in the Universe, they are laboratories for the study of the early stages of Galaxy formation (e.g. Binney \& Merrifield 1998; Freeman \& Bland-Hawthorn 2002). Each cluster is made up by an essentially simple population of stars, i.e. practically all stars were born from the same molecular cloud (e.g. Rosenberg et al. 2000) even though there is evidence for multiple populations in a few clusters. The comparison between the ratio of variable $\mathrm{M}$ stars in function of their masses for globular clusters with different metallicities, and the ratio for the galactic disc can tell us whether metallicity plays an important role at low temperatures.

The globular cluster NGC 6397 is particularly interesting in this context. NGC 6397 is a globular relatively easy to study due to its proximity. It is considered, together with NGC 6101 (M4), one of the two nearest clusters from the Sun, located at a distance $R_{\mathrm{SS}}=2.2_{-0.7}^{+0.5} \mathrm{kpc}$ (Heyl 2012) at $\alpha=17^{\mathrm{h}} 40^{\mathrm{m}} 42^{\mathrm{s}} .09$ and $\delta=-53^{\circ} 40^{\prime} 27^{\prime \prime} .6$ (J2000), with galactocentric coordinates $\ell=338.17$ and $b=-11.96$. NGC 6397 is classified as a corecollapsed cluster, predicted by theory decades ago and confirmed by observations (Trager, King \& Djorgovski 1995; Martinazzi et al. 2014). The distance moduli for NGC 6397 is $12.02 \pm 0.06$ (Hansen et al. 2007) with reddening $E(B-V)=0.18 \pm 0.02$ [Harris (1996) 2010 edition]. Another remarkable feature of NGC 6397 is its low metallicity. In fact, it is one of the lowest metallicity globular clusters known, with $[\mathrm{Fe} / \mathrm{H}]=-1.99 \pm 0.02$ (Carretta et al. 2009).

Kaluzny (1996) reported five new variable stars, including SX Phe stars and RR Lyrae variables. Kaluzny (1997) reported that few

*E-mail: elizandra.martinazzi@gmail.com (EM); kepler@if.ufrgs.br (SOK); costajes@gmail.com (JESC) variable stars are known in the globular cluster NGC 6397. They obtained light curves for seven additional discoveries, identified as close binaries, SX Phe stars and RR Lyrae variable. Kaluzny \& Thompson (2003), studied the central region of NGC 6397 and detected nine new variables stars, including one eclipsing binary, new SX Phe stars and low amplitude variables. Kaluzny et al. (2006) presented 12 new objects, of which six are periodic light curves and eclipsing binaries of unknown period again in the central region of the cluster.

Cohn et al. (2010) studied X-ray sources identified by the Chandra telescope near the centre of the cluster. In addition to studying nine cataclysmic variables (CVs) previously detected, they identified six new weak candidates for CV. Nascimbeni et al. (2012) conducted a search for variables and planetary transits in NGC 6397, exploring the images of the HST (Hubble Space Telescope)HST (Hubble Space Telescope). They analysed 5078 light curves, including a selection of 2215 cluster-member M dwarfs selected on the proper motions and reported 12 new variable stars. The great majority of the variables previously known are in the central region of the cluster.

The main objective of this work is to determine the variable star ratio as a function of mass for NGC 6397, studying if the variability is related to the metallicity.

\section{OBSERVATIONAL DATA AND DATA REDUCTION}

The images used in this work were obtained from the ESO program ID 083.D-0653(A) P.I. Barbara Castanheira. They were taken in 2009 July 27-28, with 8.4-m ESO-VLT-UT1 telescope (Very Large Telescope), using the FORS2 (FOcal Reducer and low dispersion Spectrograph) imager composed by two CCDs with a total of $2048 \times 2068$ pixels and 0.25 arcsec pixel ${ }^{-1}$ scale, with a usable portion total of $1670 \times 1677$ pixels. 


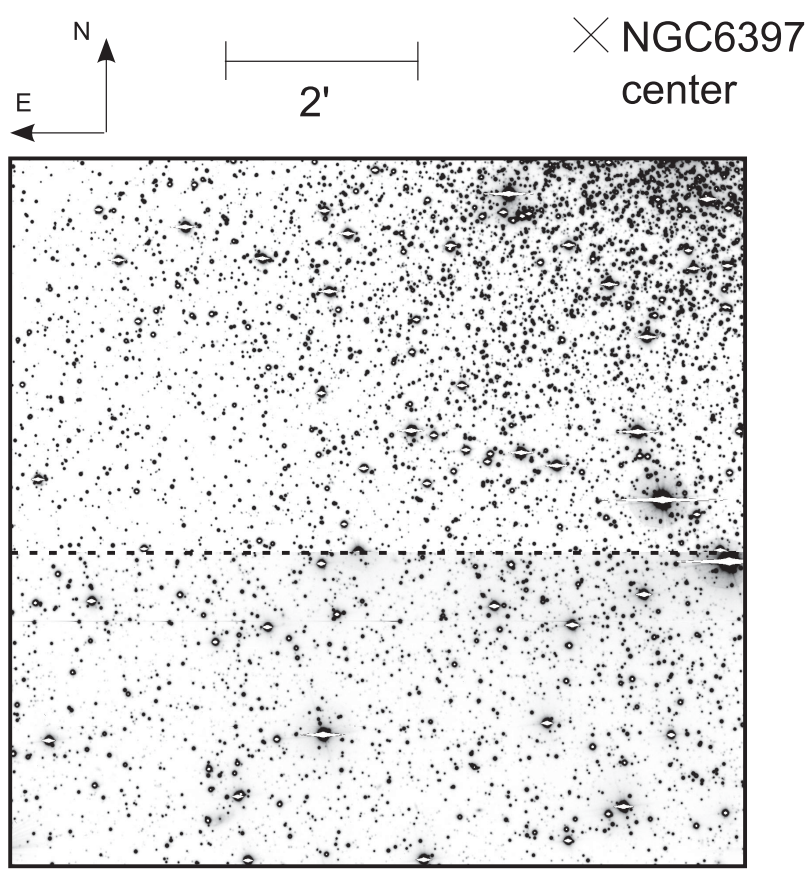

Figure 1. CCDs images of the NGC 6397, CCD1 in the top and CCD2 in the bottom. The dotted line indicates the border between both CCDs. The top edge of the right CCD1 is distant $\sim 1$ arcmin from the cluster centre ( 0 , $72 \mathrm{pc})$.

The images are taken with the filter FILT_465_250 (central wavelength $\lambda=4650 \AA$ and $\Delta \lambda=250 \AA$ ). A total of 305 images were obtained: 205 of $60 \mathrm{~s}$ on the first night, 28 of $80 \mathrm{~s}$ on the first part of the second night and 72 images of $170 \mathrm{~s}$ on the second part of the last night, starting at Julian date JD = 2455038.507350 .

We obtained $5.1 \mathrm{~h}$ of photometry on the first night and $4.8 \mathrm{~h}$ on the second night, with a separation of $19.95 \mathrm{~h}$. The CCD reading time was of the order of $30 \mathrm{~s}$. CCD1 covers a $6.8 \times 3.9 \mathrm{arcmin}^{2}$ field and CCD2 $6.8 \times 2.9 \mathrm{arcmin}^{2}$ field, amounting to $6.8 \times 6.8 \mathrm{arcmin}^{2}$ the whole field, without any gap between the CCDs. The images were centred at $\alpha=17^{\mathrm{h}} 41^{\mathrm{m}} 01.78^{\mathrm{s}}$ and $\delta=-53^{\circ} 44^{\prime} 47^{\prime \prime} .2(\mathrm{~J} 2000)$. The cluster centre, excluded from the images, is around $1.1 \mathrm{arcmin}$ to the upper right corner of the CCD1 (Fig. 1).

Image data reduction was carried out with standard IRAF routines, with tasks daofind, phot and allstar, applying Point Spread Function (PSF) photometric procedures. The astrometric calibration was done using the coordinates of the white dwarfs studied by Moehler et al. (2000). The images were centred on RA $=17 \mathrm{~h} 41 \mathrm{~min} 01,65 \mathrm{~s}$ e Dec. $=53^{\circ} 44^{\prime} 46,05^{\prime \prime}(\mathrm{J} 2000)$ to cover the region where the white dwarf candidates for DAVs were located. The correction for atmospheric extinction for a given filter was applied from the equation $m_{0}=Z P+m-K X$, where $m_{0}$ is the corrected apparent magnitude, $m$ is the apparent instrumental magnitude, $Z P$ is the zero-point, $X \simeq$ $\sec (z)$, the air mass, which varies throughout the night, depending on the zenithal distance $z$ of the object.

To create a reference magnitude data and subtract from the measured magnitudes for a given image, we compared the instrumental magnitude with the apparent magnitude of twenty standard cluster stars and determined a $Z P$ for each image. This procedure is equivalent to assuming that $Z P$ is constant and $K$ undergoes fluctuations over time. For the photometric calibration, we use the standard stars in the same field, measured by Stetson (2000) and Stetson, Catelan \& Smith (2005). After we applied the photometric corrections, we obtained the light curves of each star, where each point of the light curve is the apparent magnitude calculated photometrically by PSF fitting. The extinction coefficient for the filter FILT_465_250 was calculated from an interpolation of tabulated values. The mean value assumed for this filter was $K=0.189$. The average seeing was 1.18 arcsec for the first night and $0.69 \operatorname{arcsec}$ for the second night. We included, in the calculations, the effect of the uncertainties in the instrumental magnitude, the zero-point and the air mass.

We built the colour-magnitude diagram (CMD) shown in Fig. 2 from images obtained with $\mathrm{B}_{-} H I G H$ and $\mathrm{V}_{-} H I G H$ filters, with exposure times of $1,10,120,300 \mathrm{~s}$ each, and 1, 300 e $600 \mathrm{~s}$ with $\mathrm{U}_{-} H I G H$ filter. The data reduction was performed using IRAF routines, with the tasks daofind, phot and allstar, applying standard PSF photometric procedures. The best-fitting PSF function among those available was the elliptical Moffat with coefficient $\beta=2.5$. Objects brighter than $\mathrm{V}_{-} H I G H$ filter $\simeq 19$ were saturated in the $300 \mathrm{~s}$ images. Two lists of positions were created for each CCD using the images of $1 \mathrm{~s}$ (V_HIGH filter) for the brightnest stars and $300 \mathrm{~s}$ (V_HIGH filter) for the faint stars and both data sets were combined to avoid superposition.

In order to exclude probable non-member objects as the field stars, we used a colour-magnitude filter applied to the CMD built with the ESO-VLT data. This procedure eliminated $\sim 25$ per cent of the stars originally present in the CMD of NGC 6397. In Martinazzi et al. (2014), the luminosity and density of Milky Way field stars in the direction of NGC 6397 were estimated, obtaining a contamination by Galactic stars of 0.04 stars $\operatorname{arcsec}^{-2}$. The cut is shown in Fig. 2 at the right-hand side CMD.

We found 7617 objects in CCD1 and 3739 in CCD2, totalling 11356 objects in the whole field with magnitudes between 15 and 25. However, for stars brighter than magnitude 23 in the $465-\mathrm{nm}$ filter, we find a total 8391 stars. This range of magnitude was selected because fainter stars than magnitude 23 were contaminated by the large brightness variation of the sky (zodiacal light, even without moon) as shown in Fig. 3. We only used light curves for stars detected in more than 100 images. We also eliminated the stars near the edge of images, over a distance of 15 pixels from the edges.

\section{LOW-MASS VARIABLE STARS}

The $\mathrm{M}$ dwarfs are the most numerous stars in the Galaxy (e.g. Rojas-Ayala et al. 2014). The understanding of the inner structure of the largest population of stars in our Galaxy could occur through observations of pulsating M dwarfs. Rodríguez-López et al. (2014) presented a theoretical study of the instability strip of M dwarf stars for models in the range of $0.10-0.60 \mathrm{M}_{\odot}$, with model for different metallicity in the range of $-1.0<[\mathrm{Fe} / \mathrm{H}]<0.0$, showing theoretical evidence that solar-like oscillations can be excited in these stars. They found that the $\mathrm{M}$ dwarfs unstable modes have periods ranging from $0.014-0.46 \mathrm{~d}$ depending on the mass and evolutionary stage.

Solar-like oscillations have small amplitudes, but variations were observed to be stronger than predicted in the planning by the Kepler satellite (Gershberg 2005). Manifestations of the activity of lower main-sequence stars are numerous and diverse. Sporadic flares are observed in all layers of the stellar atmosphere, as well as cool spots involving the stellar surface and manifesting variability of largescale structures in stellar atmospheres, chromospheres and coronae (Gershberg 2005). All these effects could have longer time-scale than we studied.

Hartman et al. (2011) investigated the optical broad-band photometric variability of a sample of 27560 field $\mathrm{K}$ and M dwarfs selected by colour and proper motion using light curves from the 

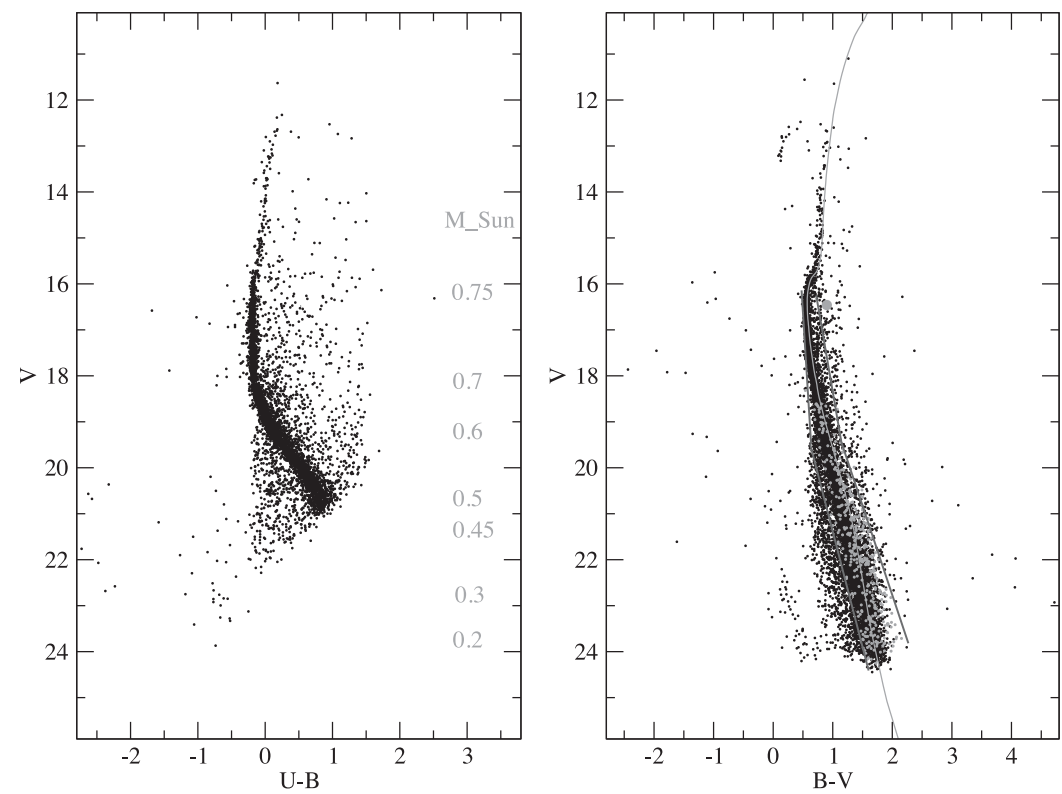

Figure 2. NGC 6397 colour-magnitude diagram for the data of this work. The values shown in the left-hand panel represent the approximate position of the masses obtained by isochrones, line in the right-hand panel, calculated by A. Dotter (2008) to the lower limit of the burning of $\mathrm{H}$. The lines at the right-hand side CMD show the colour-magnitude filter applied in order to exclude probable non-member objects as the field stars.
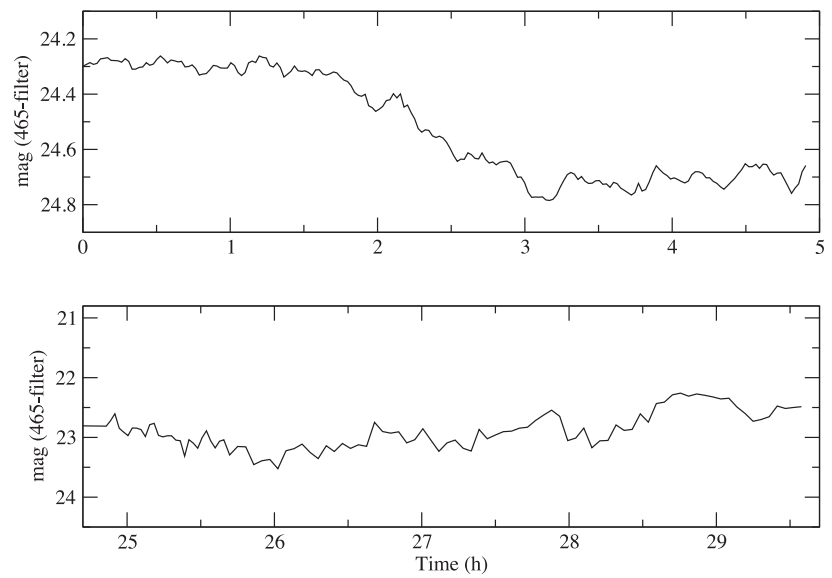

Figure 3. Brightness variation of the sky during both observation evenings in the 465-nm filter. In the first night (top panel), we used exposure time of $60 \mathrm{~s}$. In the second night (bottom panel), we used $80 \mathrm{~s}$ in the fist part and $170 \mathrm{~s}$ in second part of the observations.

HATNet survey for transiting extrasolar planets. They searched the light curves for periodic variations and for large-amplitude, longduration flare events totalling 2120 stars with potential variability using timebases between $45 \mathrm{~d}$ and $2.5 \mathrm{yr}$. They also found that the rotation periods and amplitudes of $\mathrm{K}$ and early-to mid-M dwarfs are uncorrelated for periods less than $30 \mathrm{~d}$, and that amplitude decreases with increasing rotation period greater than $30 \mathrm{~d}$. The majority of the variables are $B Y$ Dra type main-sequence stars, usually $\mathrm{K}$ or $\mathrm{M}$, exhibiting variations due to rotation coupled with star spots and other chromospheric activity. They investigate the relations between period, colour, age and activity measures, including optical flaring, for $\mathrm{K}$ and $\mathrm{M}$ dwarfs and determined that the fraction of stars that is variable with amplitudes greater than 0.01 mag and periods between 0.1 and $100 \mathrm{~d}$, increases exponentially such that $\sim 50$ per cent of field dwarf stars in the solar neighbourhood are variable at this level and at these time-scales.
Schmidt et al. (2014) presented the colours and activity of ultracool dwarfs from the Tenth Data Release of the Sloan Digital Sky Survey (SDSS) combining the previous samples of SDSS M and L dwarfs with new data obtained from the Baryon Oscillation Sky Survey (BOSS) to produce the BOSS Ultracool Dwarf sample of 11820 dwarfs. They obtained the fraction of active dwarfs rises through the M spectral sequence until it reaches $\sim 90$ per cent at spectral type L0, through the presence of $\mathrm{H} \alpha$ emission. The fraction of active dwarfs then declines to 40 per cent at spectral type M5 (mass $\sim 0.21 \mathrm{M}_{\odot}$, by the Drilling \& Landolt 2000 classification). Considering $\mathrm{M}$ dwarfs only within $100 \mathrm{pc}$ from the Galactic plane, the activity fraction increases to 10 per cent at type M0 (mass $\sim 0.51 \mathrm{M}_{\odot}$ ) and 50 per cent at type M4.

On the other hand, several studies of the low mass stars have been performed by the Kepler mission - a space telescope whose principal purpose was to detect transits and to discover exoplanets, that provides a great opportunity to study the light curves of stars with never before studied precision and coverage. In the first look at a large sample of stars with photometric data of a quality that has heretofore been only available for our Sun, Basri et al. (2010) found that nearly half of their sample was more active than the active Sun.

Newton et al. (2015) using photometry from the MEarth transit survey detected rotation periods between 0.1 and $150 \mathrm{~d}$ for 391 nearby mid-to-late $\mathrm{M}$ dwarfs in the Northern hemisphere. For fullyconvective stars with detected rotation periods, they found no correlation between metallicity and rotation period or amplitude.

Estimating M dwarf rotation periods, West et al. (2015) used photometric observations from the Earth survey for transiting exoplanets. Using spectroscopic observations and photometric light curves of 238 nearby $\mathrm{M}$ dwarfs, they examined the relationships between magnetic activity by $\mathrm{H} \alpha$ emission, rotation period and stellar age. The amplitude of the photometric modulations detected were typically 0.5 to 2 per cent peak-to-peak. For all M spectral types, they found that the presence of magnetic activity is tied to rotation, including for late-type, fully convective $\mathrm{M}$ dwarfs. 


\section{VARIABLE STARS}

For each light curve calculate the chi-square,

$\chi^{2}=\sum_{i=1}^{N}\left(\frac{m_{i}-\bar{m}}{\sigma_{i}}\right)^{2}$,

where $m_{i}$ and $\sigma_{i}$ are the $i$ th magnitude measure and its uncertainty and $\bar{m}$ is the average magnitude. Both stellar variability and underestimated uncertainties can lead to high values of $\chi^{2}$. On the other hand, overestimated uncertainties can lead to low values for $\chi^{2}$. The probability of a chi-square distribution with $(N-1)$ degrees of freedom have a value less than $\chi^{2}$ by chance can be calculated directly from the chi-square cumulative distribution function. Fig. 5 shows the calculated probabilities in function of the magnitude for the 9868 stars found in our NGC 6397 images. The Fig. 4 shows the uncertainty as function of the magnitude in the 465-nm filter, which was used in equation (1) and the Fig. 5 shows the Probability.

After sorting by the probability and $\chi^{2}$ variability method, those that showed variability in one or both the nights with amplitude variation greater than $0.2 \mathrm{mag}$ were selected. However, it was necessary to make a visual inspection of the light curve to remove false

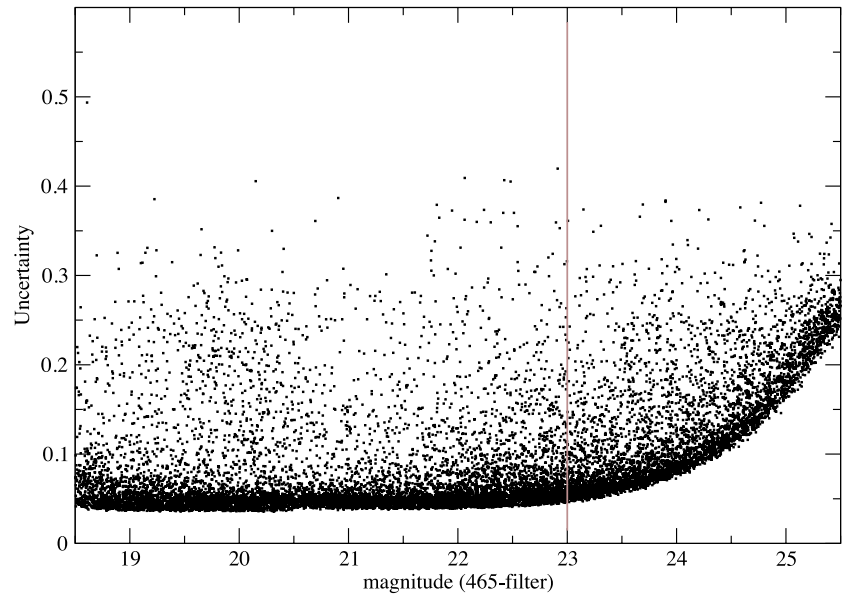

Figure 4. Uncertainty as the function of the magnitude in the 465-nm filter. The vertical line shows the magnitude limit used in this work.
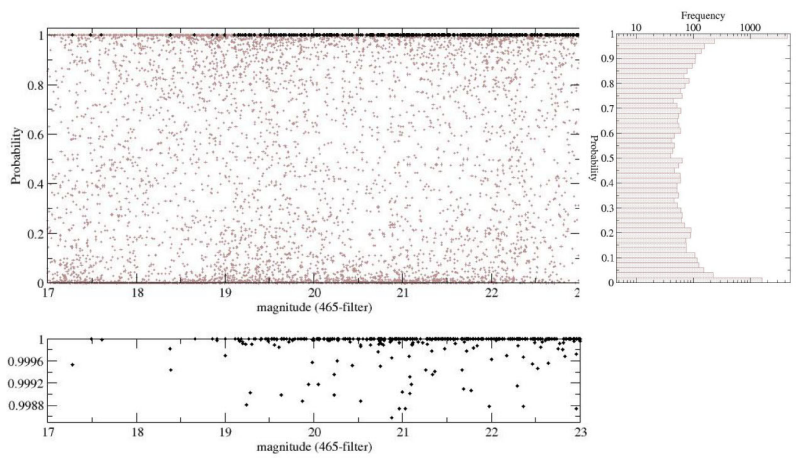

Figure 5. Distribution of probability of variability in the 465-nm filter of NGC 6397 stars. The stars selected as candidates for variables have confidence level of 99.9 per cent, with amplitudes variation greater than \pm 0.2 mag. The top shows all the 9868 stars studied. Bottom panel shows a zoom of the region with probability above 99.9 per cent, containing 412 variable stars. The right-hand panel shows frequency of the probability distribution function of the number of stars.

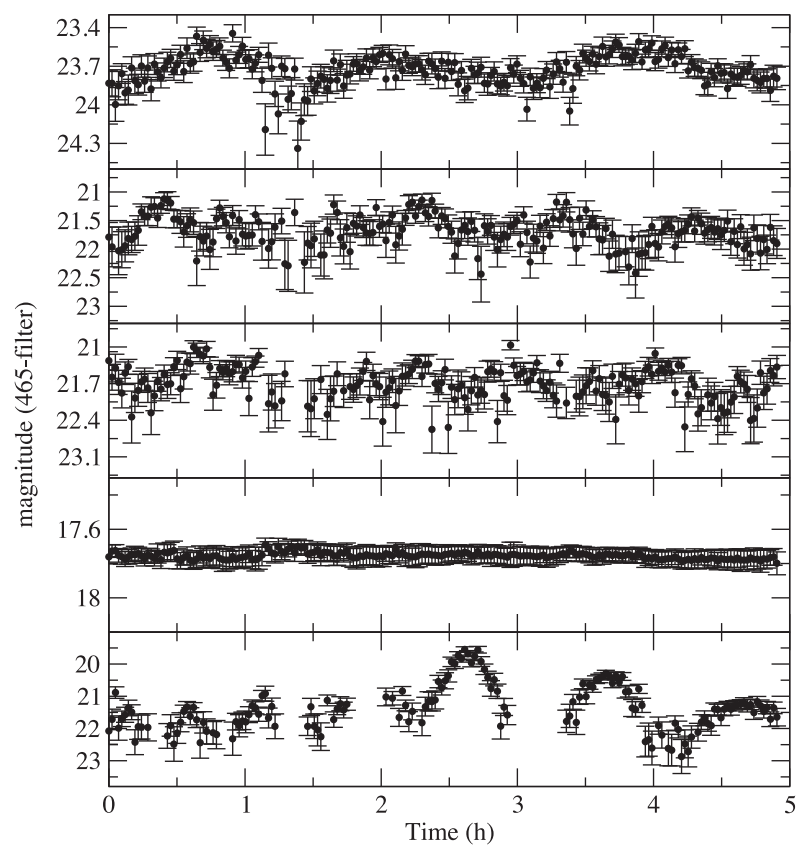

(a)

Figure 6. Panels (a), (b) and (c) are examples of light curve selected as variable stars; (d) is a typical light curve of a normal star, while (e) is an example of light curve with contamination by diffraction rays of a neighbour star brightness, eliminated by visual inspection.

alarms, discarding more than half of the candidates. Examples of candidate variables are selected stars shown in Fig. 6(a), (b) and (c). A light curve of a star considered normal or not variable, is shown in Fig. 6(d). More than half of the stars selected as candidates for variables were false alarms due to contamination of diffraction rays of bright stars, as the example shown in Fig. 6(e), or large brightness variation of the estimated background.

We found a total of 412 variables stars with 99.9 per cent confidence level, corresponding to $4.8 \pm 0.2$ per cent of the observed stars between magnitude 17 and 23 in the 465-nm filter. The objects brighter than $V \simeq 17$ were saturated, so it was not possible in this study, to search for some rapidly rotating stars, contact binaries, RR Lyrae or SX Phe stars. Fig. 7 shows the positions of variables (circles) in images obtained with the ESO-VLT.

Fig. 8 shows the histogram of not variable stars and the magnitude compared with the number of variables stars candidate and Fig. 9 shows the fraction of variables as a function of the stellar mass for NGC 6397 with magnitude between 17 and 23 (465-nm filter).

The cold stars located in the lower main sequence commonly present high chromospheric activity and will have spots on their surfaces (e.g. Valio 2012). Convection is an important form of the transport of energy and momentum in stellar interiors. For lowtemperature stars with extended convective envelope, convection exceed radiation and becomes the major form of energy transportation (e.g. Xiong et al. 2016). The active fraction peaks at spectral type M8 and drops sharply at late-M spectral types (e.g. West et al. 2004). The coronal X-ray emission levels of L dwarfs are generally below the current sensitivity limits (Stelzer et al. 2006). As the convection layer is deepening with the decreasing effective temperature the activity fraction of variability increase when the mass of stars decrease is expected. 


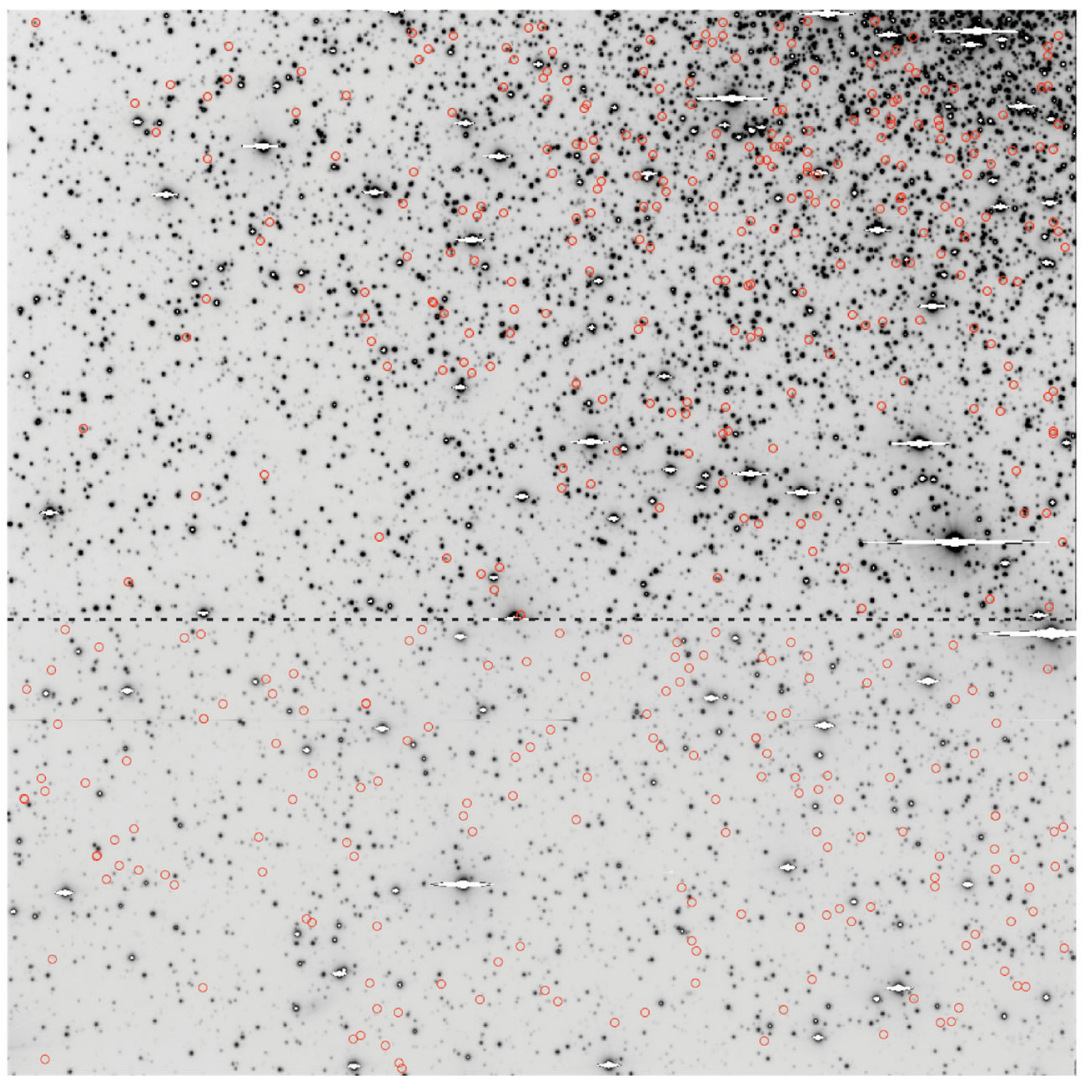

Figure 7. The circles show the positions of variables in images obtained with the ESO-VLT. Above and below CCD1 and CCD2. The dashed line shows the boundary between the two CCDs.

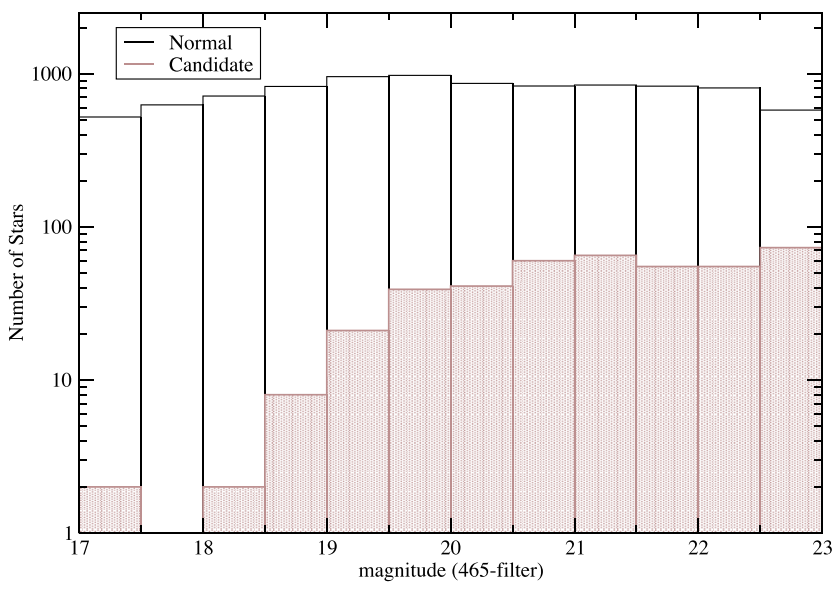

Figure 8. Magnitude distribution with the number of normal stars and magnitude with the number of variables stars candidate.

\section{DISCUSSION AND CONCLUSION}

We have conducted a photometric survey of the globular cluster NGC 6397 in a search for variable stars. We analysed 8391 light curves of NGC 6397 stars that lie in the lower part of the main sequence in the CMD, between magnitude 17 and 23 (465-nm filter) where are stars of spectral types K and M. We identified 412 stars, reaching $\sim 4.8 \pm 0.2$ per cent of variability with time-scales between 0.004 and $2 \mathrm{~d}$ with 99.9 per cent confidence level of variability to be real.

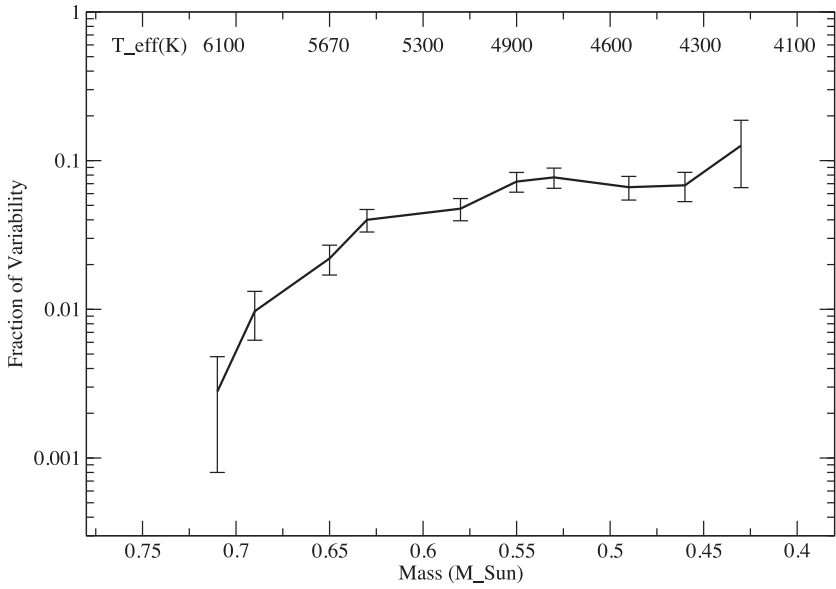

Figure 9. Fraction of candidates to variable in function of the stellar mass for NGC 6397 with magnitude between 17 and 23 (465-nm filter). The mass were estimated from the by isochrones calculated by A. Dotter (2008). The uncertainty bars are including the correction of uncertainty in completeness.

The relationship between the fraction of candidates to variables (with amplitudes variation greater than $\pm 0.2 \mathrm{mag}$ ) in function of the stellar mass of the NGC 6397 stars is shown in Fig. 9. From the curves it is observed that the fraction of the variation increases as the mass decreases. Hartman et al. (2011) also found the increased variability with decreased mass of the studied field stars. However, the stars that we observe are of low metallicity. 


\section{ACKNOWLEDGEMENTS}

We thank the referee for important comments and suggestions. We thank to ESO-VLT data program ID, support team. We acknowledge financial support from the Brazilian Institution CNPq and CAPES.

\section{REFERENCES}

Basri G. et al., 2010, ApJ, 713, L155

Binney J., Merrifield M., 1998, Galactic astronomy. Princeton, NJ

Carretta E., Bragaglia A., Gratton R., D’ Orazi V., Lucatello S., 2009, A\&A, 508,695

Cohn H. N. et al., 2010, ApJ, 722, 20

Drilling J. S., Landolt A. U., 2000, in Cox. N. A., ed. Allen's Astrophysical Quantities, 4th edn. AIP Press, New york, p. 381

Freeman K., Bland-Hawthorn J., 2002, ARA\&A, 40, 487

Gershberg R. E., 2005, Solar-Type Activity in Main-Sequence Stars. Astronomy and Astrophysics Library. Springer-Berlin, Heidelberg

Hansen B. M. S. et al., 2007, ApJ, 671, 380

Harris W. E., 1996, AJ, 112, 1487

Hartman J. D., Bakos G. Á., Noyes R. W., Sipőcz B., Kovács G., Mazeh T., Shporer A., Pál A., 2011, AJ, 141, 166

Heyl J. S. et al., 2012, ApJ, 761, 51

Kaluzny J., 1996, A\&AS, 120, 83

Kaluzny J., 1997, A\&AS, 122, 1

Kaluzny J., Thompson I. B., 2003, AJ, 125, 2534

Kaluzny J., Thompson I. B., Krzeminski W., Schwarzenberg-Czerny A., 2006, MNRAS, 365, 548

Martinazzi E., Pieres A., Kepler S. O., Costa J. E. S., Bonatto C., Bica E., 2014, MNRAS, 442, 3105

Moehler S., Heber U., Napiwotzki R., Koester D., Renzini A., 2000, A\&A, 354, L75

Nascimbeni V., Bedin L. R., Piotto G., De Marchi F., Rich R. M., 2012, A\&A, 541, A144

Newton E. R., Irwin J., Charbonneau D., Berta-Thompson Z. K., Dittmann J. A., West A. A., 2015, ApJ, 821, 93

Rodríguez-López C., MacDonald J., Amado P. J., Moya A., Mullan D., 2014, MNRAS, 438, 2371
Rojas-Ayala B., Iglesias D., Minniti D., Saito R. K., Surot F., 2014, A\&A, 571, A36

Rosenberg A., Piotto G., Saviane I., Aparicio A., 2000, A\&AS, 144, 5

Schmidt S. J., Hawley S. L., West A. A., Bochanski J. J., Davenport J. R. A., Ge J., Schneider D. P., 2015, AJ, 149, 158

Stelzer B., Micela G., Flaccomio E., Neuhäuser R., Jayawardhana R., 2006, A\&A, 448, 293

Stetson P. B., 2000, PASP, 112, 925

Stetson P. B., Catelan M., Smith H. A., 2005, PASP, 117, 1325

Trager S. C., King I. R., Djorgovski S., 1995, AJ, 109, 218

Valio A., 2012, Proc. IAU Symp. 286, Comparative Magnetic Minima: Characterizing quiet times in the Sun and StarsKluwer, Dordrecht, p. 307

West A. A. et al., 2004, ApJ, 128, 426

West A. A., Weisenburger K. L., Irwin J., Berta-Thompson Z. K., Charbonneau D., Dittmann J., Pineda J. S., 2015, ApJ, 812, 3

Xiong D. R., Deng L., Zhang C., Wang K., 2016, MNRAS, 457, 3163

\section{SUPPORTING INFORMATION}

Supplementary data are available at MNRAS online.

Table A1. Variable stars in NGC 6397 with 99.9 per cent confidence level.

Please note: Oxford University Press is not responsible for the content or functionality of any supporting materials supplied by the authors. Any queries (other than missing material) should be directed to the corresponding author for the article.

\section{APPENDIX A}

The stars identified (ID) is compared by the CCD number ( 1 or 2 ) and the ID provides by IRAF, the coordinates (RA and Dec. for J2000), reduced Chi-squere $\left[\chi^{2}\right.$ red $\left.=\chi^{2} /(\mathrm{N}-1)\right]$, the average magnitude in the B_HIGH,V_HIGH and FILT_465_250 filters of the identified variable stars in NGC 6397 are listed in Table A1. Some stars we did not get the magnitudes of some filters.

Table A1. Variable stars in NGC 6397 with 99.9 per cent confidence level. The full table is available online.

\begin{tabular}{|c|c|c|c|c|c|c|c|c|}
\hline $\begin{array}{l}\text { Star } \\
\text { ID }\end{array}$ & $\begin{array}{l}\text { RA (J2000) } \\
\text { h m s }\end{array}$ & $\begin{array}{c}\text { Dec. (J2000) } \\
0, / 1\end{array}$ & $\chi_{\mathrm{red}}^{2}$ & $\langle$ FILT_465_250 & $\left\langle\mathrm{B}_{-} H I G H\right\rangle$ & $\sigma_{\mathrm{B}}$ & $\left\langle\mathrm{V}_{-} H I G H\right\rangle$ & $\sigma_{\mathrm{V}}$ \\
\hline $1 / 103$ & $17: 40: 47.42$ & $-53: 45: 11.61$ & 1.59 & 22.81 & 23.56 & 0.05 & 22.05 & 0.03 \\
\hline $1 / 114$ & $17: 41: 02.70$ & $-53: 45: 12.39$ & 2.59 & 21.79 & 16.47 & 0.18 & 16.77 & 0.19 \\
\hline $1 / 122$ & $17: 40: 41.69$ & $-53: 45: 07.80$ & 2.57 & 21.37 & 21.37 & 0.07 & 20.43 & 0.08 \\
\hline $1 / 224$ & $17: 40: 41.69$ & $-53: 45: 07.89$ & 1.79 & 21.42 & 21.81 & 0.04 & 20.64 & 0.04 \\
\hline $1 / 322$ & 17:41:03.84 & $-53: 45: 05.49$ & 1.94 & 22.48 & 22.61 & 0.08 & 21.31 & 0.06 \\
\hline $1 / 515$ & $17: 40: 53.86$ & $-53: 44: 59.11$ & 1.55 & 19.53 & 19.81 & 0.01 & 19.01 & 0.01 \\
\hline $1 / 571$ & $17: 41: 04.40$ & $-53: 44: 58.59$ & 2.49 & 22.32 & 22.73 & 0.08 & 21.20 & 0.04 \\
\hline $1 / 651$ & $17: 41: 03.41$ & $-53: 44: 56.71$ & 1.70 & 21.70 & 21.81 & 0.07 & 20.52 & 0.10 \\
\hline $1 / 654$ & $17: 40: 48.13$ & $-53: 44: 56.57$ & 2.32 & 22.71 & 23.15 & 0.05 & 21.83 & 0.03 \\
\hline $1 / 814$ & $17: 41: 05.67$ & $-53: 44: 51.69$ & 1.63 & 22.23 & 21.57 & 0.05 & 20.69 & 0.04 \\
\hline
\end{tabular}

This paper has been typeset from a $\mathrm{T}_{\mathrm{E}} \mathrm{X} / \mathrm{LT} \mathrm{E} \mathrm{X}$ file prepared by the author. 